\title{
Practical, open source, GPU-accelerated, high-fidelity phase retrieval by simultaneous
} propagations

\author{
Jamal, Muhammad Tahir; Hansen, Anders Kragh
}

Published in:

Applied Optics

Link to article, DOI:

$10.1364 / \mathrm{AO} .402859$

Publication date:

2020

Document Version

Peer reviewed version

Link back to DTU Orbit

Citation $(A P A)$ :

Jamal, M. T., \& Hansen, A. K. (2020). Practical, open source, GPU-accelerated, high-fidelity phase retrieval by simultaneous propagations. Applied Optics, 59(34), 10761-10767. [10761]. https://doi.org/10.1364/AO.402859

\section{General rights}

Copyright and moral rights for the publications made accessible in the public portal are retained by the authors and/or other copyright owners and it is a condition of accessing publications that users recognise and abide by the legal requirements associated with these rights.

- Users may download and print one copy of any publication from the public portal for the purpose of private study or research.

- You may not further distribute the material or use it for any profit-making activity or commercial gain

- You may freely distribute the URL identifying the publication in the public portal

If you believe that this document breaches copyright please contact us providing details, and we will remove access to the work immediately and investigate your claim 


\title{
Practical, open-source, GPU accelerated, high-fidelity phase retrieval by simultaneous propagations
}

\author{
Muhammad Tahir Jamal, ${ }^{1}$ and Anders Kragh Hansen, ${ }^{2,}{ }^{*}$ \\ ${ }^{1}$ Department of Health Technology, Technical University of Denmark, Frederiksborgvej 399, 4000 Roskilde, \\ Denmark \\ ${ }^{2}$ Department of Photonics Engineering, Technical University of Denmark, Frederiksborgvej 399, 4000 \\ Roskilde, Denmark \\ *ankrh@fotonik.dtu.dk
}

\begin{abstract}
Phase retrieval is a highly useful technique that allows the calculation of the complex electric field of a beam of spatially coherent radiation based only on recordings of intensity profiles with a camera. In this work, we demonstrate a new technique for single-beam multiple-intensity phase retrieval based on simultaneous propagations that provides improved fidelity results compared to standard methods (0.9931 compared to 0.9646 ) and a $34 \mathrm{~dB}$ reduction in background noise level. The implementation is fast, open-source, user-friendly, can be run on either CPUs or GPUs and is available for download at https://gitlab.gbar.dtu.dk/biophotonics/PhaseRetrieval.
\end{abstract}

(C) 2020 Optical Society of America

\section{Introduction}

For decades, phase retrieval has been recognized as a very useful family of techniques that can be used for estimating the complex electric field of a beam of spatially coherent radiation, including the phase. While the magnitude of the field is easy to determine as the square root of the intensity, which in turn can be measured with ordinary laboratory beam cameras, the phase can only be indirectly measured using, for example, Shack-Hartmann wavefront sensors. Such sensors suffer from low resolution and as such are not able to measure the fine details in the wave front needed for the resulting field to be used as an input to further numerical simulations involving beam propagation, for example for modelling spatially dependent nonlinear interactions.

Since Gerchberg and Saxton demonstrated the first practical phase retrieval method in 1972 [1], many variants of the technique have been developed over the years $[2,3]$. They have all improved in various ways upon the original method especially in terms of convergence speed and lowering the risk of the iterative technique getting stuck in local minima. In particular, methods based on single-beam multiple-intensity reconstruction (SBMIR) [4-9], are quite successful. Unlike the original Gerchberg-Saxton method, which uses only two intensity measurements as input, the SBMIR techniques use more than two intensity measurements as inputs, thus basing the retrieval on a larger set of information and greatly aiding the convergence rate and fidelity (defined in the theory section) of the result.

In this work, we define a fidelity figure of merit and present a new SBMIR variant of the Gerchberg-Saxton technique that offers very high fidelity of the result, even in the presence of small amounts of superimposed spontaneous emission such as that from laser diodes, while maintaining a fast convergence rate. Most commonly, SBMIR techniques update the field estimate by propagating it to one plane at a time, performing an unconditional substitution of the field magnitude before propagating to the next plane.

The novelty of this work is that we combine (i) the conditional magnitude substitution introduced in [8] with (ii) a scheme in which the field estimate is propagated simultaneously, rather than sequentially, to all measurement planes and (iii) adaptive $x-y$ shifting of the measurement frames 
to correct position uncertainties of the measurements.

We also emphasize some important details of the implementation that heighten the impact of this work, specifically (i) that we have made the code available open-source, (ii) it is written in user-friendly MATLAB using mex functions that combine the speed of code written in the $C$ programming language with the versatility and familiarity of MATLAB, (iii) it supports optional acceleration on Nvidia Graphics Processing Units (GPUs), and (iv) it is bundled with an optional script to extract suitable input data from data saved from Spiricon M2-200s profilers or similar.

We demonstrate the usefulness of the technique by characterizing the nontrivial wave front of the emission from a special tapered master oscillator power amplifier (MOPA) laser diode device in which the light exits at a non-normal angle to the facet. Knowing the phase of such a beam can be used to determine the distortions caused by the facet angle and for fabricating an optical phase mask for correcting for the distortions, or for modelling spatially dependent frequency conversion of the light in nonlinear crystals.

\section{Algorithm}

Throughout this article, the method we use for numerically propagating a laser beam's complex electric field from one plane to another is that of Fresnel diffraction [10], which is valid in both the Fresnel and Fraunhofer diffraction regimes. Unlike, e.g., finite-difference beam propagation methods (FD-BPM) which require many computation steps to cover long distances, Fresnel diffraction propagation can cover arbitrarily long distances in a single computation step, making it efficient. As explained in [11], the underlying Fresnel approximation is generally considered equivalent to the paraxial approximation, which is well satisfied for the beams considered in this work, where the beam divergence angles are on the order of $5 \mathrm{mrad}$. According to the Fresnel approximation to the propagation integral, the field $E_{2}$ in plane 2 can be calculated from the field $E_{1}$ in plane 1 as

$$
E_{2}(x, y)=\mathcal{F}^{-1}\left\{\mathcal{F}\left\{E_{1}(x, y)\right\} \exp \left(i \Delta z\left[k-\pi \lambda\left(f_{x}^{2}+f_{y}^{2}\right)\right]\right)\right\}
$$

where $\mathcal{F}$ denotes the Fourier transform, $i$ is the imaginary unit, $\Delta z$ is the difference in the planes' positions, $k$ is the angular wavenumber, $\lambda$ is the wavelength and $f_{x}$ and $f_{y}$ are the spatial frequency components.

The method demonstrated in this work is an SBMIR method. This means that a beam of spatially coherent light propagating in free space along the $z$-axis is focused and $N$ measurements of intensity profiles are made at various values of defocus. These measurements thus preferably show the beam profiles in the near field of the focus, the far field and the planes in between. This set of measurements is similar to those one would take to determine the beam quality factor $M^{2}$ of a beam. We denote by $I_{j}$ the measured intensity profile in plane $j$ normalized to an integral of 1 .

In our method of phase retrieval, we then define a reference plane at an arbitrary choice of $z$-position, which need not coincide with any measurement, and denote the running field estimate in the reference plane $E_{0}$. A diagram of the algorithm is shown in Figure 1, and it proceeds as follows:

1. Initialize an ansatz field $E_{0}$ in the reference plane with a magnitude equal to $\sqrt{I_{j}}$, where $j$ is the index of the measurement plane closest to the chosen reference plane. The phase can be either zero everywhere or initialized with random phase in each pixel.

2. Multiply $E_{0}$ with a real gain factor $g$ and propagate this amplified reference plane to all of the measurement planes to obtain the set of $N$ different fields $E_{j}=\mathcal{F}^{-1}\left\{\mathcal{F}\left\{g E_{0}\right\} \exp (i \Delta z[k-\right.$ $\left.\left.\left.\pi \lambda\left(f_{x}^{2}+f_{y}^{2}\right)\right]\right)\right\}$, where $\Delta z$ depends on $j$. The gain factor functions analogously to that of a laser gain medium, increasing the magnitude without changing the phase, and is constant throughout the process. 
3. For each plane and for each pixel, perform a conditional substitution of magnitude to obtain what we call the attenuated field $A_{j}:\left|A_{j}(x, y)\right|=\min \left(\left|E_{j}(x, y)\right|, \sqrt{I_{j}(x, y)}\right)$. This means that if the field estimate $E_{j}$ in a pixel has magnitude less than the square root of the measured intensity, the field in that pixel is not attenuated. The phase of $A_{j}$ is set equal to the phase of $E_{j}$.

4. Propagate all of the attenuated fields $A_{j}$ back to the reference plane and obtain the new field estimate $E_{0}$ in the reference plane as the mean of all the back-propagated fields: $E_{0}=\frac{1}{N} \sum_{j=1}^{N} \mathcal{F}^{-1}\left\{\mathcal{F}\left\{A_{j}\right\} \exp \left(-i \Delta z\left[k-\pi \lambda\left(f_{x}^{2}+f_{y}^{2}\right)\right]\right)\right\}$.

5. Repeat steps 2 - 4 iteratively until convergence, defined as the root mean square of changes in the normalized E-field amplitudes dropping below $10^{-6}$.

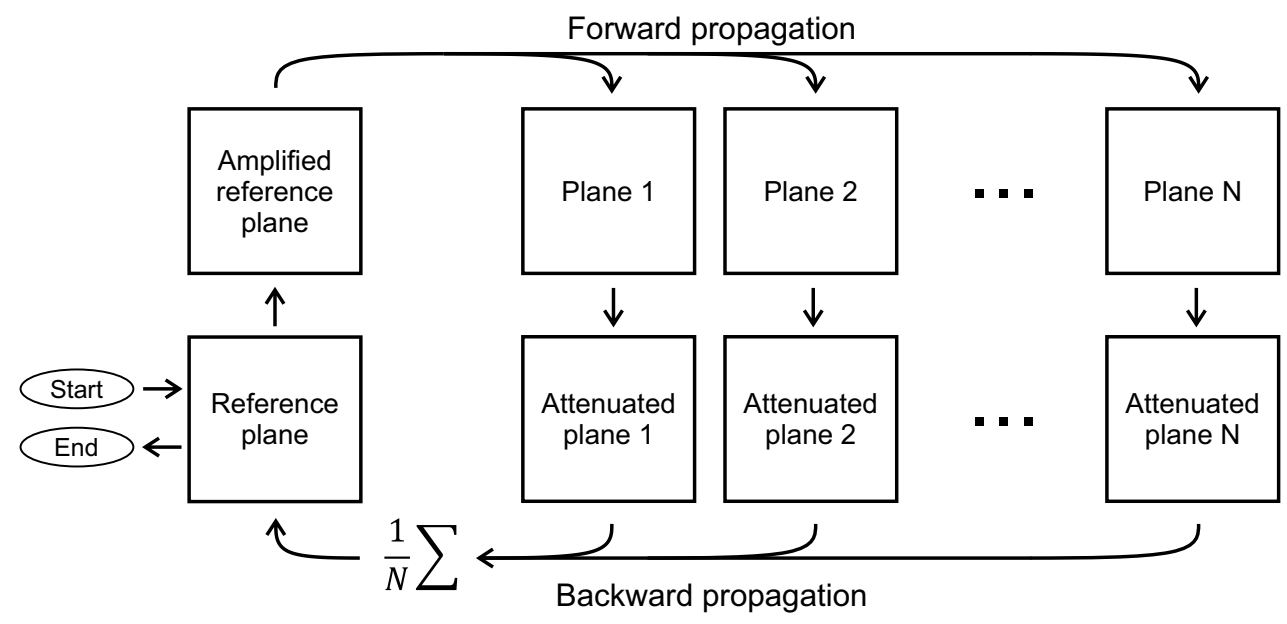

Fig. 1. A diagram of the method introduced in this paper. Each square box represents a complex electric field. "Propagation" refers to Fresnel diffraction using Fourier transforms.

The gain factor $g$ must be greater than 1 to counteract the attenuation imposed in step 3 . Empirically, a small $g$ yields a result with relatively lower noise, while greater values of $g$ result in the successful retrieval of more fine features in the beam profile, up to a certain limit. The choice of $g$ will be studied in the Characterization section.

In the above, we omitted the description of the adaptive $x-y$ shifting. While performing an iteration, this adaptive $x-y$ shifting can optionally be inserted just after step 2: Calculate the power (sum of squared norms of the field pixels) of a hypothetical $A_{j}$ as it would be calculated in step 3 in the absence of any $x-y$ shift of $I_{j}$, and compare this power to those achieved by shifting $I_{j}$ positively or negatively in the $x$ and $y$ directions. The $x$ and $y$ shifts that result in the highest power of the hypothetical resulting $A_{j}$, that is, the lowest overall attenuation, are the new accepted $x$ and $y$ shifts of that frame. This condition is nearly equivalent to that of the locating the maximum cross correlation coefficient, but empirically provided better results. Step 3 then proceeds with the correspondingly shifted measured intensity profile. These shifts are cumulative. To reduce computation time in our implementation, this adaptive $x-y$ shifting is only performed every 5 iterations, only up to and including iteration 50 and only shifts of up to \pm 2 pixels along each dimension are checked every time.

Several of the features of the algorithm deserve some justification: 
a. The conditional magnitude substitution in step 3 is similar to that of [8], and serves to better handle detector noise and cases in which small amounts of superimposed spontaneous emission is co-propagating with the beam, which will often be the case for emission from some lasers such as laser diodes. The reason for this is that noise or incoherent light causes non-zero intensity measurements at spatial locations where the actual coherent part of the laser field is essentially zero, for example $(x, y)$ locations far from the beam or at local destructive interference within the beam. Such pixels are associated with a poorly defined phase. In the traditional Gerchberg-Saxton method or most other SBMIR methods, performing a full magnitude substitution in such pixels will amplify what is essentially a beam component with a random, non-physical phase, leading to poor fidelity (defined below) of the result.

b. The gain factor $g$ is a consequence of the fact that the conditional magnitude substitution can only attenuate and not amplify the field. Therefore, this power loss has to be counteracted by a gain.

c. The adaptive $x-y$ shifting described above is necessary to compensate for experimental uncertainties in the $x$ and $y$ positions of each measurement. For example, for a measurement series taken by moving a camera along a translation stage, non-linear movement of the stage would result in measurements with different $x$ and $y$ offsets. If these shifts are not corrected, the result will have comparatively worse fidelity.

As a quantitative description of the degree to which the propagated result of the phase retrieval corresponds to the intensity measurements, we define the fidelity for each measured frame in the following way: First, calculate the matrix of cross correlation coefficients $c_{j}(k, l)$ between the measured intensity profile $I_{j}$, normalized to the sum of the squares of its pixel values, and the intensity profile calculated by numerically propagating the retrieved field to that plane, $\left|E_{j}\right|^{2}$. Per definition, these coefficients are given by

$$
c_{j}(k, l)=\sum_{i_{x}=0}^{N_{x}-1} \sum_{i_{y}=0}^{N_{y}-1} \frac{I_{j}\left(i_{x} \Delta x, i_{y} \Delta y\right)}{\sum_{x, y} I_{j}^{2}}\left|E_{j}\left(\left(i_{x}-k\right) \Delta x,\left(i_{y}-l\right) \Delta y\right)\right|^{2},
$$

where $N_{x}$ and $N_{y}$ are the number of pixels in the $x$ and $y$ directions and $i_{x}$ and $i_{y}$ are index variables. The fidelity is then defined as the maximum value of $\left|c_{j}(k, l)\right|^{2}$. In other words, the fidelity of each frame is the best achievable overlap integral between the measured and calculated intensity profiles among all the different $x-y$ shifts one may apply to the measurement. The normalization applied ensures that if the phase retrieved field corresponds exactly to the measured intensity profile, the fidelity is 1 .

\section{Characterization}

In this section, we will demonstrate the performance of the method using the data set that is included with the code in the git repository at https://gitlab.gbar.dtu.dk/biophotonics/PhaseRetrieval. We generated this data set from the laser beam of a distributed Bragg-reflector tapered diode laser emitting spatially coherent single-frequency light at a wavelength of $1064 \mathrm{~nm}$, that was then frequency doubled to $532 \mathrm{~nm}$ in a nonlinear crystal. The beam was collimated in the vertical direction with an aspheric lens and in the horizontal direction with a cylindrical lens. We then directed the beam into a Spiricon M2-200s laser profiler that consists of some filters, a lens with focal length $300 \mathrm{~mm}$, a delay line on a translation stage and a camera. We made intensity measurements in 38 planes with this profiler, with lens-camera distances $z$ ranging from $210 \mathrm{~mm}$ to $470 \mathrm{~mm}$. Neglecting slight ellipticity, the beam full-widths at half-maximum were roughly 
$300 \mu \mathrm{m}$ at $z=210 \mathrm{~mm}, 90 \mu \mathrm{m}$ in the focus at $z=300 \mathrm{~mm}$ and $400 \mu \mathrm{m}$ at $z=470 \mathrm{~mm}$. The images were recorded at a resolution of $1600 \times 1200$ but downsampled to $800 \times 600$ for the characterizations of this section, and the pixel pitch was $4.4 \mu \mathrm{m}$ in both the $x$ and $y$ directions. The signal to noise ratio of the measurements, defined as the peak intensity value divided by the root mean square of background pixel values, was roughly 550 in the raw measurements and 1100 in the downsampled measurements.

\subsection{The fidelity figure of merit}

To help the reader interpret the numerical values of the fidelities that we will provide in the rest of this section, we have plotted three arbitrary examples of calculated intensity distributions on a logarithmic scale in Figure 2 along with the corresponding measured intensity and we list the fidelity of each example. Of course, different applications will require different fidelities, but subjectively one sees a very good agreement between the calculated and measured intensity profiles at a fidelity of 0.986 , at which all beam features above the $-30 \mathrm{~dB}$ noise floor of the measurement also appear in the calculated intensity profile.
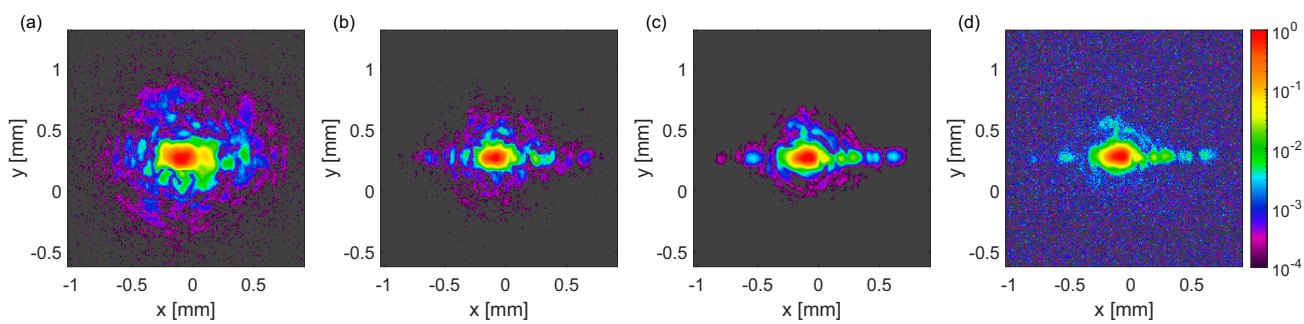

Fig. 2. Illustration of the fidelity figure of merit. The plots show the normalized intensity on a logarithmic color scale for 3 arbitrary examples of phase retrieval results (a - c) and the corresponding measured intensity profile (d) in that plane. The color bar of (d) applies to all plots. The fidelities are (a) 0.923, (b) 0.971, and (c) 0.986. A high-fidelity result such as (c) reproduces the fine features of the measurement best, but even a comparatively low fidelity result such as (a) correctly reproduces certain beam properties such as the $x$ and $y$ widths at half-maximum. The above profiles were obtained by terminating the algorithm before the convergence criterion was met.

\subsection{Choice of gain factor}

The main free variable with regards to the input is the gain factor $g$, applied in step 2 of the method. In general the optimal value of $g$ should be expected to depend on the noise of the input measurements, the number of input measurements and their $z$-positions, and how fine the beam features the user wants to retrieve. Therefore, the user must try different values of $g$ and select the one that works best, either in terms of fidelity, background noise, or whatever other criterion the user may have. As an example, we ran the phase retrieval on the test data set of 38 measurements with four different choices of gain factor. The resulting intensity profiles at the focal plane are shown in Figure 3. The number of iterations to obtain convergence is nearly the same for all choices of $g$.

\subsection{How many measurements are needed?}

As with all other SBMIR techniques, a central question when using it is how many measurements are necessary. To find out, we chose various subsets of measurements out of the full set of 38 and performed the phase retrieval on only those measurements. In all cases, we still calculated the fidelities of all 38 frames regardless of which subset we chose for phase retrieval. The fidelities 

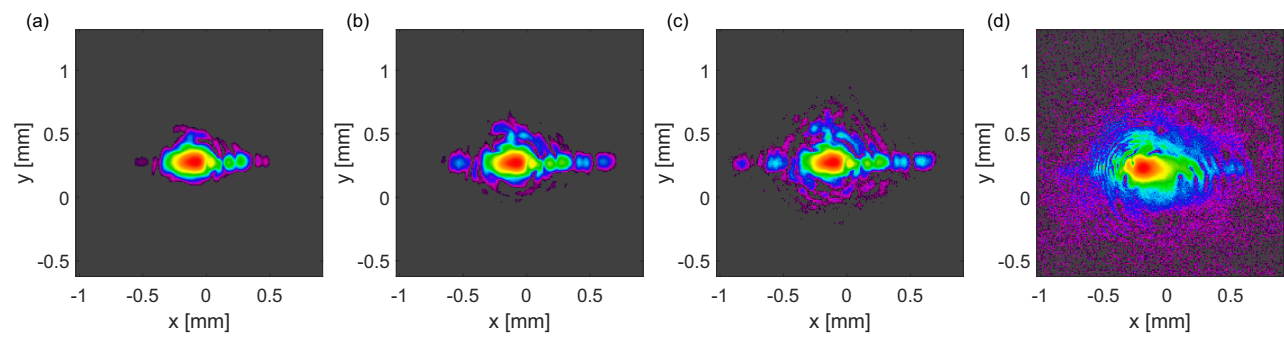

Fig. 3. Illustration of the normalized phase retrieved intensity profiles on a logarithmic color scale at $z=300 \mathrm{~mm}$ for various choices of the gain factor $g$; (a) 1.01, (b) 1.1, (c) 1.4, and (d) 1.8. The color scale and the measured intensity is that of Figure 2(d). In $(a-c)$, it is clear that a higher gain factor enables the retrieval of finer beam structure features. In (d), the gain factor is too high.

at convergence for four different choices of subsets are plotted in Figure 4. This analysis shows that, with this particular set of test data, 6 measurements were sufficient. For each approach, the oversampling ratio given by the number of measurements $N_{x} N_{y} N_{f}$ divided by the number of unknowns $2 N_{x} N_{y}$ is $\frac{N_{f}}{2}$, where $N_{x}$ and $N_{y}$ are the pixel resolutions of the camera images and $N_{f}$ is the number of frames involved. The factor of 2 comes from the fact that the retrieved E-field is complex, while the inputs are real.

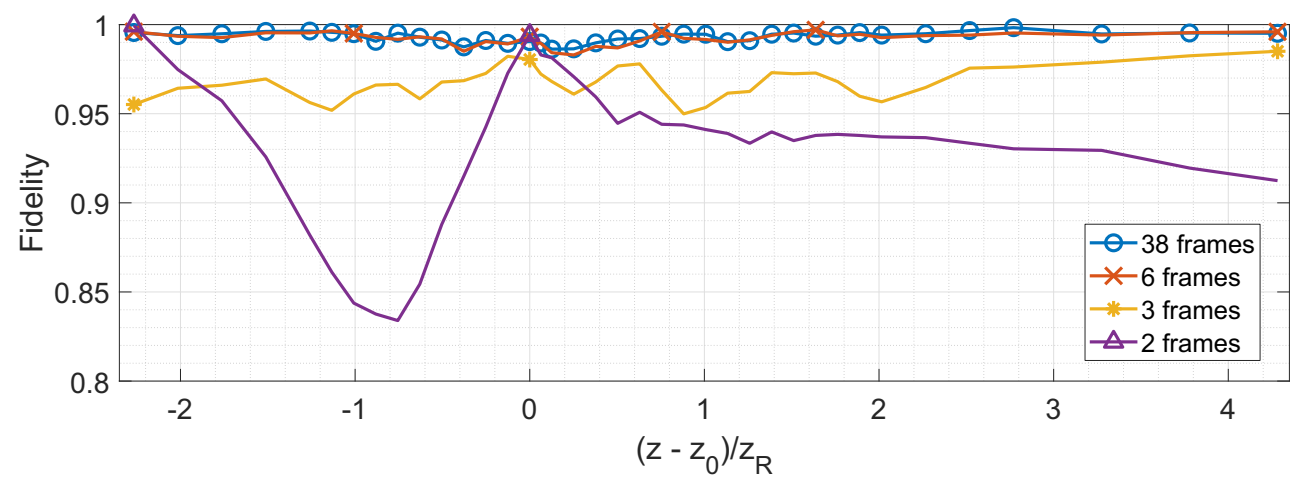

Fig. 4. Illustration of fidelities at convergence for four different choices of the subset of measurements (frames) to involve in the phase retrieval process. $z_{0}$ : Focus $z$-position, $z_{R}$ : Rayleigh length, $3.97 \mathrm{~cm}$. For each choice, markers are shown only on those frames that were involved in the phase retrieval. Involving more than 6 frames offered, in this case, no significant additional benefit. Involving only 3 frames resulted in markedly poorer fidelity. Involving only two frames, such as in the original Gerchberg-Saxton method, results in high fidelity only for the two involved frames, while the fidelities in all other planes are much lower.

\subsection{Comparison to standard SBMIR}

To get a sense of how the method compares to other phase retrieval methods, we have also implemented the basic SBMIR method of reference [4] and used it on the same data set as above. As the authors of that work observed, the basic SBMIR method only reaches convergence (root mean square of change in normalized E field amplitude $<10^{-6}$ ) for artificially generated inputs without noise and otherwise is just terminated once stagnation sets in. We compare in Figure 5 
the results both in terms of the achieved fidelities and the standard deviation $\sigma$ of background noise. (1): The method of this work, (2): SBMIR of reference [4], without frame shifting, (3): Same as (2) but with frame shifting, as described in the algorithm section.
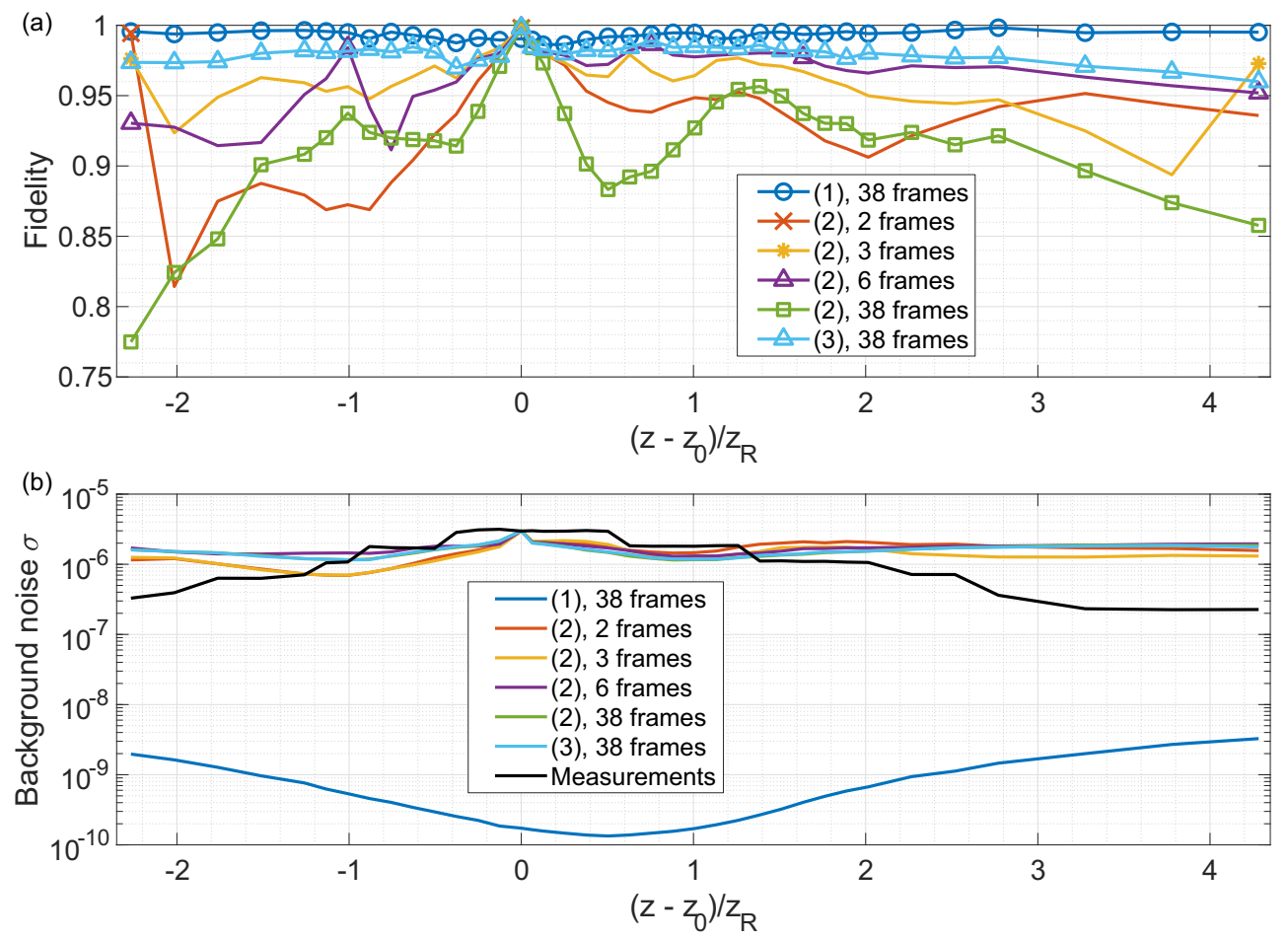

Fig. 5. Comparison of (a) the fidelity and (b) the background noise of the method presented in this work to prior work. The $z$ axis is defined as in Figure 4. See the text for details. The best results with methods (1), (2) and (3) have mean fidelities of 0.9931, 0.9646 and 0.9799 , respectively, while the background noise standard deviations for the camera measurements and the frames retrieved by methods (1), (2) and (3) are $1.5 \cdot 10^{-6}, 6.9 \cdot 10^{-10}, 1.7 \cdot 10^{-6}$ and $1.6 \cdot 10^{-6}$, respectively.

Note that when involving 38 frames in the phase retrieval, method (2) neither converged nor stagnated, since the convergence criterion above alternatingly increased and decreased with the number of iterations. The plotted result of (2) with 38 frames is a snapshot of the nonconverged and nonstagnated E-field after 4 hours of iterations. The frame at the focus has been used as the first and final frame for the methods (2) and (3), which is why the fidelity of that frame is unity. The method proposed in this work, method (1), yields the highest mean fidelity as well as a background noise level $34 \mathrm{~dB}$ below that of the input measurements and the results of methods (2) and (3).

\subsection{Computation speed}

Another important quality of the technique and its implementation is the high speed, which is partly due to its implementation as MATLAB mex functions and due to the optional GPU acceleration. The GPU acceleration is implemented using Nvidia's Compute Unified Device Architecture (CUDA) and thus requires an Nvidia graphics card. For speed testing, we used a test PC with an Intel i5 9400F processor and a GeForce RTX 2070 graphics card. For comparison purposes we also wrote an all-MATLAB implementation of the technique, that is, with no use of 
mex functions or GPU acceleration.

We first compared execution speeds using the 6-frame subset of the test data used throughout this section shown in the red curve of Figure 4. The all-MATLAB implementation took $118 \mathrm{~s}$ to obtain convergence, while the CPU mex implementation took $45.4 \mathrm{~s}, 2.6 \times$ faster. The GPU mex implementation took $15.3 \mathrm{~s}$ for the same calculations, a speedup of $3.0 \times$ compared to the CPU mex implementation or $7.7 \times$ compared to the all-MATLAB implementation. The number of iterations necessary to obtain convergence was 226.

When we performed phase retrieval on the full 38 -frame set, the CPU mex implementation took $88.7 \mathrm{~s}$ and the GPU mex implementation took $26.2 \mathrm{~s}$. The number of iterations needed for convergence was 124. Evidently, involving more frames in the phase retrieval makes each iteration take longer due to the larger quantity of data, but this is partly offset by requiring fewer iterations to reach convergence.

Note that for phase retrieval in a time-critical application, one could use a lower resolution and/or a less strict convergence criterion, making the process even faster.

\section{Demonstration on "bent" tapered laser diode}

As a demonstration of the usefulness of the technique, we show here a phase retrieval on the non-trivial beam emitted by an unusual MOPA laser diode device. This type of laser device is described in [12] and can emit up to 9.5 watts of single-frequency light at a wavelength of $1067 \mathrm{~nm}$ under standard operating conditions. In addition to the trivial ellipticity and astigmatism that is typical of tapered laser diodes and can be easily corrected, this sort of device also exhibits a special type of beam distortion caused by the non-zero angle between the center axis of the tapered section and the facet normal.

For the measurements, the device was mounted on a conduction cooled package (CCP) mount which was temperature stabilized at $25^{\circ} \mathrm{C}$. The current through the MO, control and PA sections were $300 \mathrm{~mA}, 10 \mathrm{~mA}$ and $4 \mathrm{~A}$, respectively, resulting in $1.15 \mathrm{~W}$ of output. Since a fast axis collimation of such non-trivial sources with standard microlenses can easily introduce aberrations, we tried performing the collimation with two different lenses; an A414 Rochester aspheric lens (focal length $3.3 \mathrm{~mm}, 0.47 \mathrm{NA}$ ) and an Olympus LMPlan IR objective lens (3.6 mm focal length, $50 \times / 0.55$ NA, infinity corrected). Simulations in the ray tracing software BeamXpertDESIGNER indicated that the A414 lens would introduce negligible aberrations for a source of this type, and the objective lens is a high quality lens designed for IR microscopy, so we expected that it, too, would introduce only negligible aberrations. Both choices of lens resulted in collimated beams of identical appearance, so we concluded that both lenses were indeed suitable for aberration-free fast axis collimation and proceeded with the Olympus objective lens.

Downstream of the fast axis collimation lens mentioned above, we placed a cylinder lens of focal length $25 \mathrm{~mm}$ to collimate the slow axis, after which we attenuated the beam by reflecting it off two uncoated glass wedges. We then sent the beam into the same Spiricon M2-200s beam profiler used for the data of the previous section and recorded measurements in 31 planes from $z=240 \mathrm{~mm}$ to $z=630 \mathrm{~mm}$.

The measurements made in the first plane, the focal plane and the last plane are shown in Figure $6(\mathrm{a}-\mathrm{c})$ to illustrate the non-trivial propagation of the beam. Although the beam has an appearance very similar to that of one collimated by an off-axis collimation lens, we verified thoroughly that the collimation lens was indeed correctly centered. We used our method of phase retrieval with a gain factor of 1.4, converging after 1224 iterations, achieving final minimum, mean and maximum fidelities among all the frames of $0.9902,0.9938$ and 0.9961 , respectively. The intensity profiles of the phase retrieved result in the planes of the three example measurements are shown in Figure $6(\mathrm{~d}-\mathrm{f})$ and the corresponding phase profiles in Figure 6(g - i ). The full-resolution phase retrieved complex $E$-field data is available from the corresponding author upon request. 

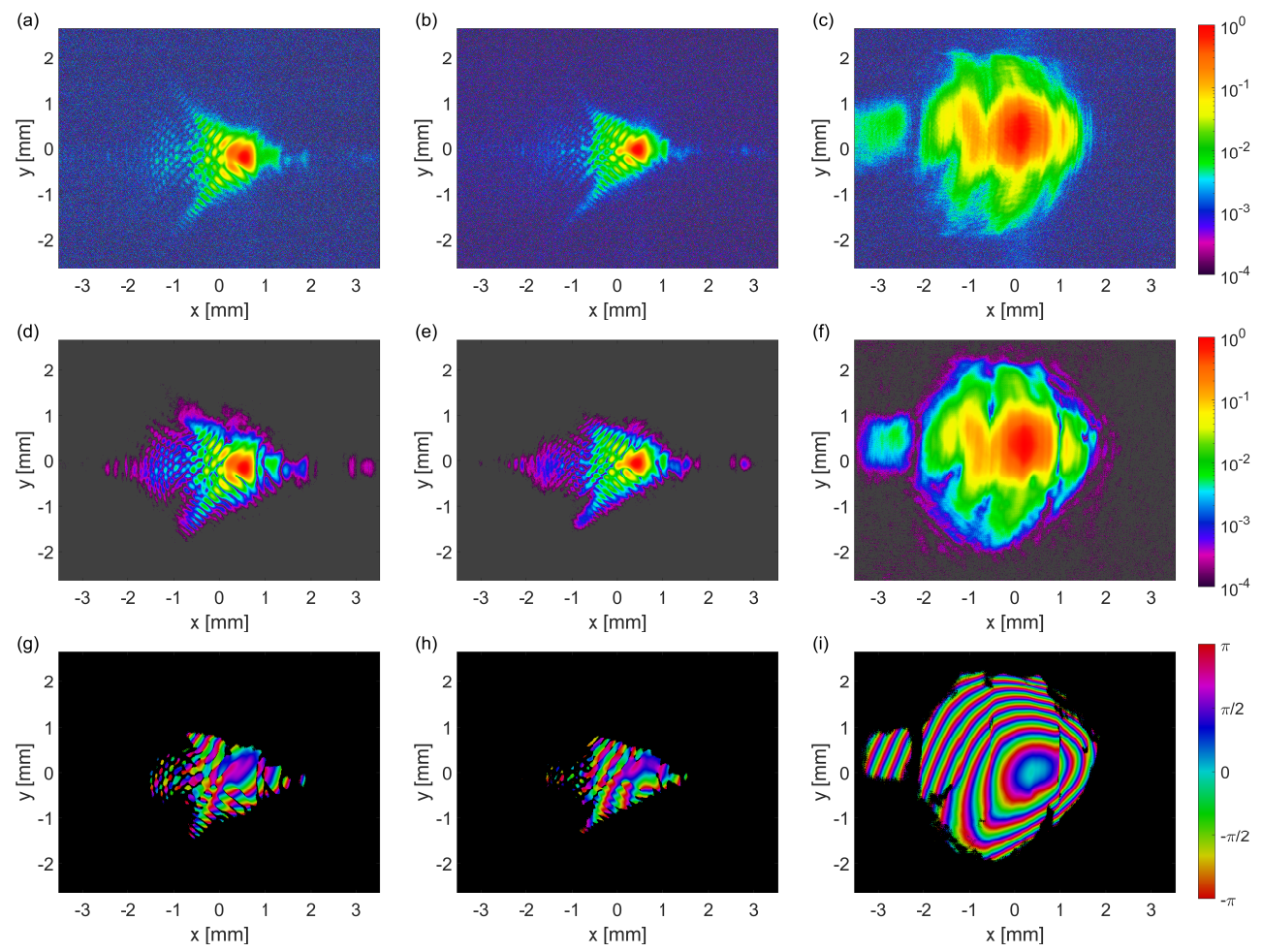

Fig. 6. Measurements in the first $(\mathrm{a}, z=240 \mathrm{~mm})$, focal $(\mathrm{b}, z=300 \mathrm{~mm})$ and last $(\mathrm{c}, z=640 \mathrm{~mm})$ planes of the data set for phase retrieval of the MOPA source. Corresponding phase retrieval intensity profiles are shown in (d-f) and phase profiles in (g-i). Excellent agreement is found between the retrieved and measured intensity profiles, even though the beam exhibits complicated structure in the weak side lobes.

\section{Conclusion}

We have demonstrated that the new method of phase retrieval provides high-fidelity results that are well suited for numerical propagation to different planes. The implementation as MATLAB mex functions combined the versatility and familiarity of MATLAB with the speed of code written in the $\mathrm{C}$ programming language, offering a significant speedup. Additionally, the optional GPU-accelerated implementation offers an additional speedup, which could be relevant for time-critical applications. The fact that the code is user-friendly, free and open-source ensures that other laser engineers and researchers can easily download and use the code themselves.

\section{Funding}

This work was supported by the European Commission within the project FBI (721766).

\section{Acknowledgments}

We are very thankful to Dr. Christof Zink and the rest of the group of Prof. Bernd Sumpf at the Ferdinand-Braun-Institut in Berlin for providing both of the laser sources used in this work.

\section{Disclosures}

The authors declare no conflicts of interest. 


\section{References}

1. R. W. Gerchberg and W. O. Saxton, "A practical algorithm for the determination of phase from image and diffraction plane pictures," Optik 35, 237-246 (1972).

2. J. R. Fienup, "Phase retrieval algorithms: a comparison,” Appl. Opt. 21, 2758 (1982).

3. Y. Shechtman, Y. C. Eldar, O. Cohen, H. N. Chapman, J. Miao, and M. Segev, "Phase Retrieval with Application to Optical Imaging: A contemporary overview," IEEE Signal Process. Mag. 32, 87-109 (2015).

4. L. Allen and M. Oxley, "Phase retrieval from series of images obtained by defocus variation," Opt. Commun. 199, 65-75 (2001).

5. P. F. Almoro, A. M. S. Maallo, and S. G. Hanson, "Fast-convergent algorithm for speckle-based phase retrieval and a design for dynamic wavefront sensing," Appl. Opt. 48, 1485 (2009).

6. K. Falaggis, T. Kozacki, and M. Kujawinska, "Accelerated single-beam wavefront reconstruction techniques based on relaxation and multiresolution strategies," Opt. Lett. 38, 1660 (2013).

7. J. F. Binamira and P. F. Almoro, "Accelerated single-beam multiple-intensity reconstruction using unordered propagations," Opt. Lett. 44, 3130 (2019).

8. A. K. Hansen, "Coherent laser phase retrieval in the presence of measurement imperfections and incoherent light," Appl. Opt. 56, 7341 (2017).

9. R. Yazdani and H. Fallah, "Wavefront sensing for a Shack-Hartmann sensor using phase retrieval based on a sequence of intensity patterns," Appl. Opt. 56, 1358 (2017)

10. D. Voels, Computational Fourier Optics, a Matlab Tutorial (SPIE Press, 2011).

11. J. W. Goodman, Introduction to Fourier Optics, 3rd ed. (Roberts \& Co. Publishers, 2005).

12. C. Zink, A. Maabdorf, J. Fricke, P. Ressel, B. Sumpf, G. Erbert, and G. Trankle, "Monolithic Master Oscillator Tilted Tapered Power Amplifier Emitting 9.5 W at 1060 nm,” IEEE Photonics Technol. Lett. 32, 59-62 (2020). 